\title{
Effect of Surface Properties on Wrinkling of Ultrathin Films
}

\author{
Rui Huang ${ }^{1}$; Christopher M. Stafford ${ }^{2}$; and Bryan D. Vogt ${ }^{3}$
}

\begin{abstract}
Thin films bonded to compliant substrates often develop wrinkles when subjected to an applied or inherent compressive stress. This paper presents a bilayer model to account for surface effects on the wrinkling of ultrathin solid films. Assuming a surface layer of finite thickness, effects of surface properties on the critical strain, the equilibrium wavelength, and the wrinkle amplitude are discussed in comparison with conventional analysis. We apply this model to explain experimental observations of wrinkling in thin polymer films. The measured wrinkle wavelengths and amplitudes deviate from conventional analysis for ultrathin films with thickness less than $30 \mathrm{~nm}$. The bilayer model provides a consistent understanding of the observed deviations, based on which a set of material properties for the surface and the bulk of the polymer films are deduced from the experimental data.
\end{abstract}

DOI: 10.1061/(ASCE)0893-1321(2007)20:1(38)

CE Database subject headings: Models; Aerospace engineering; Compressive strength; Polymers; Material properties.

\section{Introduction}

Modern technology has developed a broad range of applications with integrated hard and soft materials at micron to nanometer scales. Typically, wrinkling of thin films has been treated as a nuisance to be avoided (Iacopi et al. 2003; Yin et al. 2003) rather than an exquisite pattern to be exploited. However, once understood, it is possible to control and even use wrinkling, for example, to develop stretchable interconnects for flexible electronics (Watanabe et al. 2002; Lacour et al. 2003), tunable diffraction gratings (Harrison et al. 2004), force spectroscopy in cells (Harris et al. 1980), biocompatible cell and particle alignment (Efimenko et al. 2005), and advanced metrology methods (Stafford et al. 2004). Mechanics was established long ago for wrinkling of elastic faces in structural sandwich panels (Allen 1969) and has been revisited recently for nonlinear analysis (Groenewold 2001; Huang et al. 2005) and inelastic behavior (Im and Huang 2004; Huang 2005) in thin-film structures. It has been understood that wrinkling is a result of stress-driven instability. For an elastic film on an elastic substrate, a critical compressive stress exists, beyond which a stable equilibrium wrinkled state can be determined from an energetic analysis that accounts for elastic strain energy in the film and the substrate. Surface energy is considered negligible in the conventional analysis. However, as

\footnotetext{
${ }^{1}$ Assistant Professor, Dept. of Aerospace Engineering and Engineering Mechanics, Univ. of Texas, Austin, TX 78712 (corresponding author). E-mail: ruihuang@mail.utexas.edu

${ }^{2}$ Staff Scientist, Polymers Division, National Institute of Standards and Technology, Gaithersburg, MD 20899.

${ }^{3}$ Assistant Professor, Dept. of Chemical Engineering, Arizona State Univ., Tempe, AZ 85287; formerly, Postdoctoral Fellow, National Institute of Standards and Technology, Gaithersburg, MD 20899.

Note. Discussion open until June 1, 2007. Separate discussions must be submitted for individual papers. To extend the closing date by one month, a written request must be filed with the ASCE Managing Editor. The manuscript for this paper was submitted for review and possible publication on August 26, 2005; approved on February 22, 2006. This paper is part of the Journal of Aerospace Engineering, Vol. 20, No. 1, January 1, 2007. CASCE, ISSN 0893-1321/2007/1-38-44/\$25.00.
}

the film thickness reaches nanometer scale, it is expected that surface energy as well as other surface effects will become important (Huang and Suo 2003).

Recently, a wrinkle-based metrology was developed to measure elastic properties of thin polymer films (Stafford et al. 2004). For ultrathin polymer films (thickness less than $30 \mathrm{~nm}$ ), the measured wrinkle wavelengths deviate from the conventional solution, and the deduced elastic modulus of the films decreases with decreasing film thickness (Stafford et al. 2006). In addition, the measured wrinkle amplitudes also differ significantly from the conventional analysis. These experimental results raise a fundamental question as to what are the physical origins for the deviations, in particular, the thickness dependence of the deduced elastic modulus in the ultrathin films. This paper proposes a bilayer model to account for surface effect on wrinkling that explains such a change in modulus. In the next section, the model is developed and compared to the conventional analysis. The third section briefly describes the experiments with thin polymer films. The experimental results are discussed and compared to the model predictions in the following section. The final section concludes with a summary of the findings.

\section{Analysis of Wrinkling}

Consider a thin elastic film of thickness $h$ bonded to a thick elastic substrate. Subjected to a uniaxial compressive strain $\varepsilon$ parallel to the interface, the film has a propensity to buckle. Due to the constraint of the substrate, two types of buckling modes may occur: If the substrate is stiff and the interface is relatively weak (e.g., due to contamination), the film may delaminate and buckle away from the substrate (Hutchinson and Suo 1992; Ortiz and Gioia 1997). Alternatively, if the substrate is compliant, the film may buckle without delamination, as the substrate undergoes conformal deformation. This paper focuses on the latter case, which is often called wrinkling. Fig. 1 schematically illustrates the two buckling modes. Following a conventional linear stability analysis (Allen 1969), the critical compressive strain for wrinkling is 

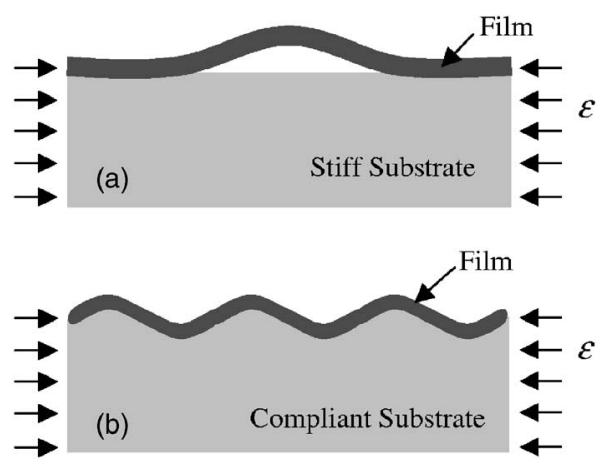

Fig. 1. Two buckling modes in thin films: (a) buckle-delamination; (b) wrinkling

$$
\varepsilon_{c}=-\frac{1}{4}\left(\frac{3 \bar{E}_{s}}{\bar{E}_{f}}\right)^{2 / 3}
$$

where $\bar{E}=E /\left(1-v^{2}\right)=$ plane-strain modulus with the subscripts $f$ and $s$ for the film and the substrate, respectively. The negative sign denotes compression. When $\varepsilon<\varepsilon_{c}$ (larger magnitude in compression), a bifurcation occurs and the stable equilibrium state of the film wrinkles in a sinusoidal form with a wavelength $\lambda$ and an amplitude $A$ as given by (Huang et al. 2005)

$$
\begin{gathered}
\lambda=2 \pi h\left(\frac{\bar{E}_{f}}{3 \bar{E}_{s}}\right)^{1 / 3} \\
A=h \sqrt{\frac{\varepsilon}{\varepsilon_{c}}-1}
\end{gathered}
$$

The previous results are obtained as a consequence of competition between elastic strain energy in the film and the substrate. Specifically, wrinkling relaxes the compression-induced strain energy in the film, but leads to an increase in bending energy in the film and strain energy in the substrate. In these calculations, surface energy is completely neglected. For ultrathin films with thickness at the nanometer scale, however, surface energy could be significant enough to compete with the strain energy and change the stability condition as well as the equilibrium state. A simple comparison between typical surface energy density of solids $\left(\approx 1 \mathrm{~J} / \mathrm{m}^{2}\right)$ and strain energy density $\left(\approx 10^{7} \mathrm{~J} / \mathrm{m}^{3}\right)$ suggests that the surface energy could become important for film thickness in the order of $100 \mathrm{~nm}\left(10^{-7} \mathrm{~m}\right)$ or less.

During wrinkling, the surface of the film is strained, but with no mass exchange between the surface and the bulk. It was found that, for such cases, it is the surface stress that is relevant for the stability of the film (Huang and Suo 2003). For this reason, it is important to note the difference between surface energy and surface stress for solids (Cahn 1980; Rice and Chuang 1981; Spaepen 1996; Freund 1998). Following Cahn (1980), we define the surface energy density of a solid referring to the undeformed state

$$
\gamma=\gamma_{0}+f \varepsilon
$$

where $\gamma_{0}=$ strain energy density of the corresponding undeformed surface; $\varepsilon=$ in-plane strain; and $f=$ surface stress. Thus, surface stress of a solid represents the dependence of surface energy density on mechanical deformation to the first order, which can be either positive (tensile) or negative (compressive) (Cammarata 1994; Ibach 1997). This is fundamentally different from surface energy or surface tension of a liquid, as first pointed out by Gibbs (1878).

As shown in a previous study (Huang and Suo 2003), accounting for the surface energy given by Eq. (4) in a stability analysis is equivalent to defining an effective membrane force in the conventional analysis, which includes contributions from stress in the bulk of the film and the surface stress, namely

$$
N=\bar{E}_{f} h \varepsilon+f
$$

Therefore, comparing the two terms on the right-hand side of Eq. (5) dictates when the contribution of surface stress would be important. Typically, it happens for film thickness less than $100 \mathrm{~nm}$. With the effect of surface stress, the critical strain for wrinkling becomes

$$
\varepsilon_{c}=-\frac{1}{4}\left(\frac{3 \bar{E}_{s}}{\bar{E}_{f}}\right)^{2 / 3}-\eta
$$

where $\eta=f / \bar{E}_{f} h=$ dimensionless number characterizing the effect of surface stress. Eq. (6) dictates that a tensile surface stress $(f, \eta>0)$ leads to a larger magnitude for the critical strain, thus stabilizing the film from wrinkling, while a compressive surface stress $(f, \eta<0)$ destabilizes the film. For typical values of elastic modulus and surface stress, the number $\eta$ is negligibly small for relatively thick films, but becomes significant for ultrathin films. Beyond the critical strain, the wrinkle amplitude at the equilibrium state becomes

$$
A=h \sqrt{\frac{\varepsilon+\eta}{\varepsilon_{c}+\eta}-1}
$$

On the other hand, the wrinkle wavelength [Eq. (2)] is independent of the strain or membrane force and is thus unaffected by surface stress. Therefore, including surface stress alone cannot explain the observed deviation in wavelengths for ultrathin polymer films.

Next we develop a bilayer model to account for surface effects. It is well known that atomic or molecular structure at a surface is different from bulk structure in solids. As a result, many material properties (e.g., mass density, electrical conductivity, elastic modulus, etc.) at the surface differ from their counterparts in the bulk. Such difference is negligible for large structures. For nanostructures, however, the surface-to-volume ratio is large, and surface effects can be significant. For thin solid films of interest in this study, one may assume that there exists a surface layer of different elastic modulus in each film. Compared to the bulk elastic modulus, the surface elastic modulus can be either greater (hard surface) or smaller (soft surface), depending on the material and processing conditions. The thickness of the surface layer may vary from about one atomic spacing for crystalline materials (Ibach 1997) to a few nanometers for polymers (Mansfield and Theodorou 1991). In addition, a residual stress or strain may exist in the surface layer, which can be considered equivalent to uniformly distributing the surface stress (in the unit of force per length) into the surface layer of a finite thickness. Therefore, the originally single-layer homogeneous film becomes a bilayer composite film with the same total thickness, and the effect of surface energy or surface stress is accounted for by the presence of the surface layer. As will be shown later, this bilayer model is able to consistently explain both the wavelengths and the amplitudes in the wrinkling experiments of ultrathin polymer films. 
The effective modulus of a bilayer film can be obtained by the rule of mixture. For in-plane stretching/compression, the effective modulus is

$$
\bar{E}_{f, \text { stretching }}=\bar{E}_{f}\left(1-\frac{\delta}{h}\right)+\bar{E}_{f}^{*}\left(\frac{\delta}{h}\right)
$$

where $\delta=$ thickness of the surface layer $(\delta<h)$ and $\bar{E}_{f}^{*}=$ surface elastic modulus. For bending, the effective modulus is

$$
\begin{aligned}
\bar{E}_{f, \text { bending }}= & \bar{E}_{f}\left(1-\frac{\delta}{h}\right)^{3}+\bar{E}_{f}^{*}\left(\frac{\delta}{h}\right)^{3}+3 \bar{E}_{f}\left(1-\frac{\delta}{h}\right)\left(1-\xi-\frac{\delta}{h}\right)^{2} \\
& +3 \bar{E}_{f}^{*}\left(\frac{\delta}{h}\right)\left(2-\xi-\frac{\delta}{h}\right)^{2}
\end{aligned}
$$

where

$$
\xi=\frac{\bar{E}_{f}\left(1-\frac{\delta}{h}\right)^{2}+\bar{E}_{f}^{*}\left(\frac{\delta}{h}\right)\left(2-\frac{\delta}{h}\right)}{\bar{E}_{f}\left(1-\frac{\delta}{h}\right)+\bar{E}_{f}^{*}\left(\frac{\delta}{h}\right)}
$$

The two effective moduli are slightly different, leading to a somewhat complicated analysis of wrinkling. Nevertheless, the same approach as the conventional analysis can be applied. First, the total membrane force in the bilayer film becomes

$$
N_{\text {bilayer }}=\bar{E}_{f, \text { stretching }} h \varepsilon+f
$$

where the residual stress in the surface layer is taken to be $f / \delta$ to account for the effect of surface stress $f$. The critical membrane force for wrinkling is controlled by the bending stiffness of the film, namely

$$
N_{c, \text { bilayer }}=-\frac{3^{2 / 3}}{4} \bar{E}_{f, \text { bending }}^{1 / 3} \bar{E}_{s}^{2 / 3} h
$$

Equating Eqs. (11) and (12) determines the critical strain

$$
\varepsilon_{c, \text { bilayer }}=-\frac{3^{2 / 3}}{4} \frac{\bar{E}_{f, \text { bending }}^{1 / 3} \bar{E}_{s}^{2 / 3}}{\bar{E}_{f, \text { stretching }}}-\frac{f}{\bar{E}_{f, \text { stretching }} h}
$$

Eq. (13) is similar to Eq. (6) except for the difference in the bending and stretching moduli for bilayer films.

The equilibrium wrinkle wavelength also depends on the bending stiffness, namely

$$
\lambda_{\text {bilayer }}=2 \pi h\left(\frac{\bar{E}_{f, \text { bending }}}{3 \bar{E}_{s}}\right)^{1 / 3}
$$

On the other hand, the wrinkle amplitude depends on both bending and stretching

$$
A_{\text {bilayer }}=h \sqrt{\left(\frac{N_{\text {bilayer }}}{N_{c, \text { bilayer }}}-1\right) \frac{\bar{E}_{f, \text { bending }}}{\bar{E}_{f \text {,stretching }}}}
$$

The difference between Eqs. (13)-(15) and Eqs. (1)-(3) will become transparent by comparing them graphically. Fig. 2 illustrates the wrinkle wavelength as a function of film thickness. For a homogeneous film $\left(\bar{E}_{f}^{*}=\bar{E}_{f}\right.$ or $\left.\delta=0\right)$, Eq. (14) reduces to Eq. (2), and the wavelength-to-thickness ratio is a constant, i.e., the

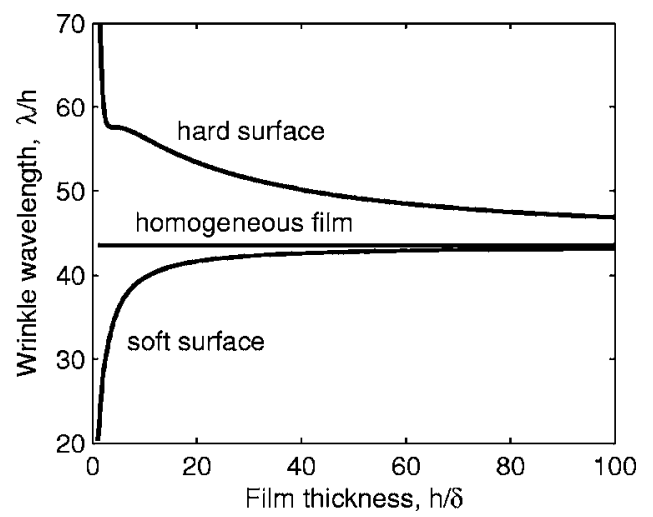

Fig. 2. Wrinkle wavelength as a function of film thickness for a homogeneous film $\left(\bar{E}_{f} / \bar{E}_{s}=1,000\right)$ in comparison with bilayer films with a soft surface $\left(\bar{E}_{f}^{*} / \bar{E}_{f}=0.1\right)$ and a hard surface $\left(\bar{E}_{f}^{*} / \bar{E}_{f}=10\right)$, respectively

wrinkle wavelength is linearly proportional to the film thickness. For a bilayer film, however, the wavelength-to-thickness ratio is not a constant, especially when the ratio $h / \delta$ is small. If the surface modulus is smaller (i.e., soft surface), the wavelength decreases as the film thickness decreases. If the surface modulus is greater (i.e., hard surface), the wavelength increases. For both cases, the wavelength approaches to that for a homogeneous film as the film thickness becomes much larger than the thickness of the surface layer; the approaching is slower for the hard surface case. The ratio $h / \delta$ essentially represents the ratio between the number of atoms in the surface layer and that in the bulk, which determines the significance of the surface effect. The introduction of a length scale $\delta$ leads to a size effect for the wrinkle wavelength. Using the conventional solution in Eq. (2) to directly extract film modulus from measured wrinkle wavelengths would lead to a thickness-dependent modulus, as will be shown later for the polymer thin films.

Fig. 3 plots the wrinkle amplitude as a function of film thickness, subjected to a constant strain $(\varepsilon=-0.02)$. The trend is similar to that for wavelength in Fig. 2. The amplitude is linearly

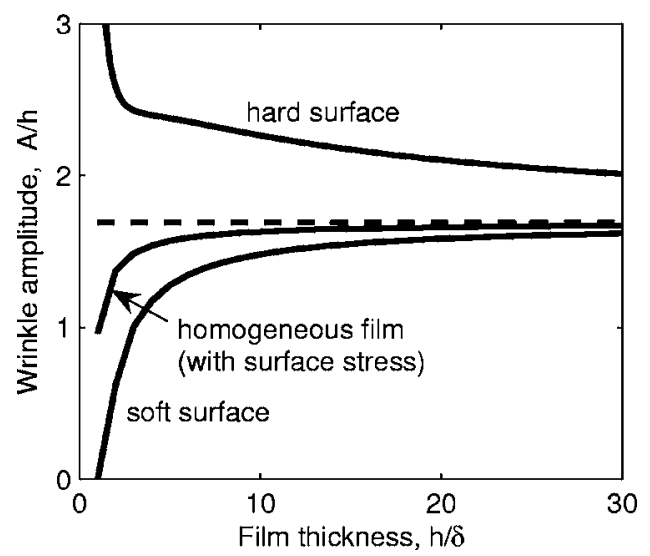

Fig. 3. Wrinkle amplitude as a function of film thickness subjected to a constant strain $(\varepsilon=-0.02)$, for a homogeneous film $\left(\bar{E}_{f} / \bar{E}_{s}=1,000\right.$, $\left.f=10 \bar{E}_{s} \delta\right)$ in comparison with bilayer films with a soft surface $\left(\bar{E}_{f}^{*} / \bar{E}_{f}=0.1\right)$ and a hard surface $\left(\bar{E}_{f}^{*} / \bar{E}_{f}=10\right)$, respectively. The dashed line corresponds to a homogeneous film with no surface stress effect $(f=0)$. 


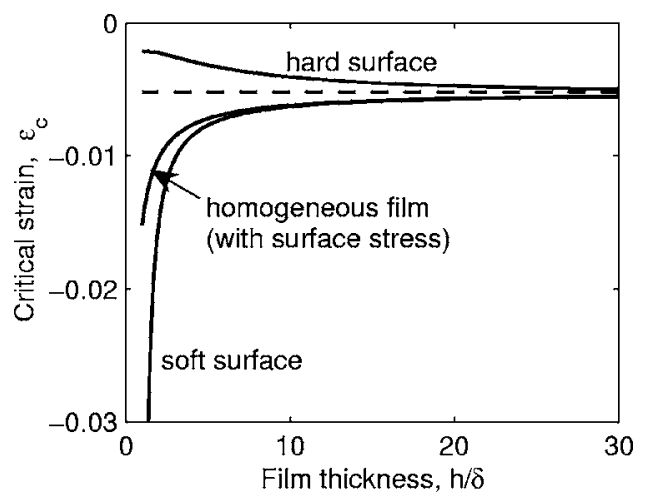

Fig. 4. Critical strain for wrinkling as a function of film thickness. The dashed line is from conventional analysis for homogeneous films with no surface stress effect.

proportional to film thickness for a homogeneous film without considering the effect of surface stress. With a positive (tensile) surface stress $(f>0)$, the amplitude-to-thickness ratio decreases as the film thickness decreases [Eq. (7)]. The effect is significant for ultrathin films. Further, with a hard surface layer, the film is effectively stiffer and the average stress is higher under the constant strain, which tends to increase the wrinkle amplitude. The competition between the stabilizing effect of a tensile surface stress and the destabilizing effect of a hard surface may lead to a nonmonotonic dependence of the amplitude-to-thickness ratio on film thickness. In this particular case $\left(\bar{E}_{f}^{*} / \bar{E}_{f}=10\right.$ and $\left.f=10 \bar{E}_{s} \delta\right)$, however, the stiffness effect dominates the surface stress effect, leading to a monotonic increase of the amplitude-to-thickness ratio as the film thickness decreases. On the other hand, with a soft surface layer, both the stiffness effect and the surface stress effect tend to lower the amplitude; thus the amplitude-tothickness ratio monotonically decreases as film thickness decreases, eventually becoming zero at a critical thickness. This critical thickness can be determined from Eq. (13) by equalizing the critical strain to the applied strain. Below the critical thickness, the applied strain is lower in magnitude than the critical strain (i.e., $|\varepsilon|<\left|\varepsilon_{c}\right|$ ); thus the film is stable with zero wrinkle amplitude. The critical strain itself is a function of film thickness as shown in Fig. 4. It can be seen from Eq. (13) that a soft surface layer leads to an effectively softer film, requiring larger compressive strain to cause wrinkling. A hard surface layer, on the other hand, reduces the critical strain. The surface stress adds an additional term, which increases the critical strain if it is tensile and decreases otherwise. The magnitude of the critical strain increases rapidly as film thickness decreases for ultrathin films with a soft surface and a tensile surface stress. Note that the critical strain predicted by the conventional analysis [homogeneous film with no surface stress effect; Eq. (1)] is independent of film thickness (dashed line in Fig. 4).

\section{Experiments}

The experimental details of the study of wrinkling in ultrathin polymer films can be found elsewhere (Stafford et al. 2004, 2005). We highlight here necessary details to benefit and illuminate our discussion. Thin films of polystyrene (PS) were spin coated from dilute toluene solutions onto polished silicon wafers. Film thickness was varied from 200 to $5 \mathrm{~nm}$ as determined by $\mathrm{X}$-ray reflectivity. Films were then transferred onto polydimethyl-

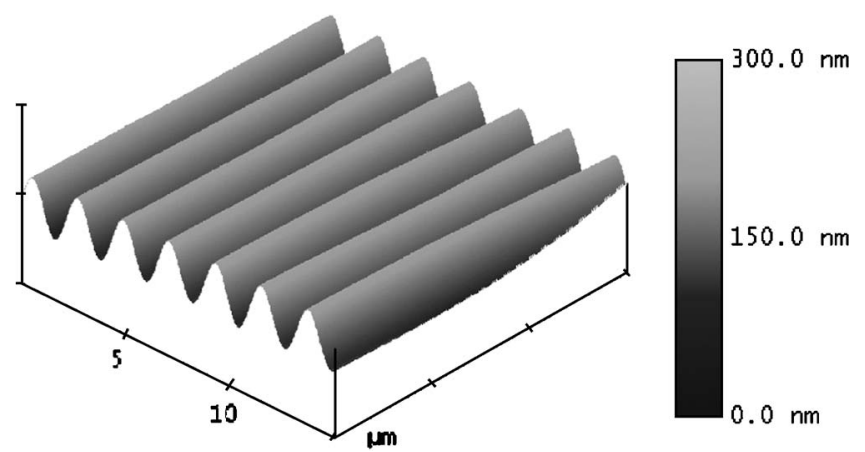

Fig. 5. AFM height image of wrinkling in a PS film $\left(M_{w}=1,800 \mathrm{~kg} / \mathrm{mol}, h=40.4 \mathrm{~nm}\right)$ on a PDMS substrate subjected to a $2.5 \%$ compressive strain. The scan area is $15 \mu \mathrm{m} \times 15 \mu \mathrm{m}$ and the height scale is $300 \mathrm{~nm}$.

siloxane (PDMS) substrates and mounted to a home-built apparatus (Stafford et al. 2005) in order to apply a uniaxial compressive strain. The magnitude of the strain was constant (2.5\%) for all films in this study. Wrinkling of the PS films was imaged by tapping mode atomic force microscopy (AFM), from which the wavelengths and amplitudes were measured. Fig. 5 shows a representative AFM height image of wrinkling in a PS film having a thickness of $40.4 \mathrm{~nm}$. Fig. 6 shows the measured wrinkle wavelength as a function of film thickness. Two different molecular masses of PS were examined $\left(M_{w}=114 \mathrm{~kg} / \mathrm{mol}\right.$ and $1,800 \mathrm{~kg} / \mathrm{mol})$, both well above the entanglement molecular mass of PS $\left(M_{e}=19 \mathrm{~kg} / \mathrm{mol}\right)$. Fig. 7 shows the wrinkle amplitude as a function of film thickness for the PS films with higher molecular mass. The elastic modulus of the PDMS substrate was measured beforehand using a traditional tensile test and was found to be 1.33 MPa for the substrate of the lower molecular mass PS films and $1.75 \mathrm{MPa}$ for the substrate of the higher molecular mass PS films. Annealing the film for several hours prior to transfer was found to have no influence on the measured wavelength. It was also confirmed that the wrinkles persist over days with no evidence of further evolution, indicating the elastic behavior for the film and the substrate. Although the applied strain is relatively

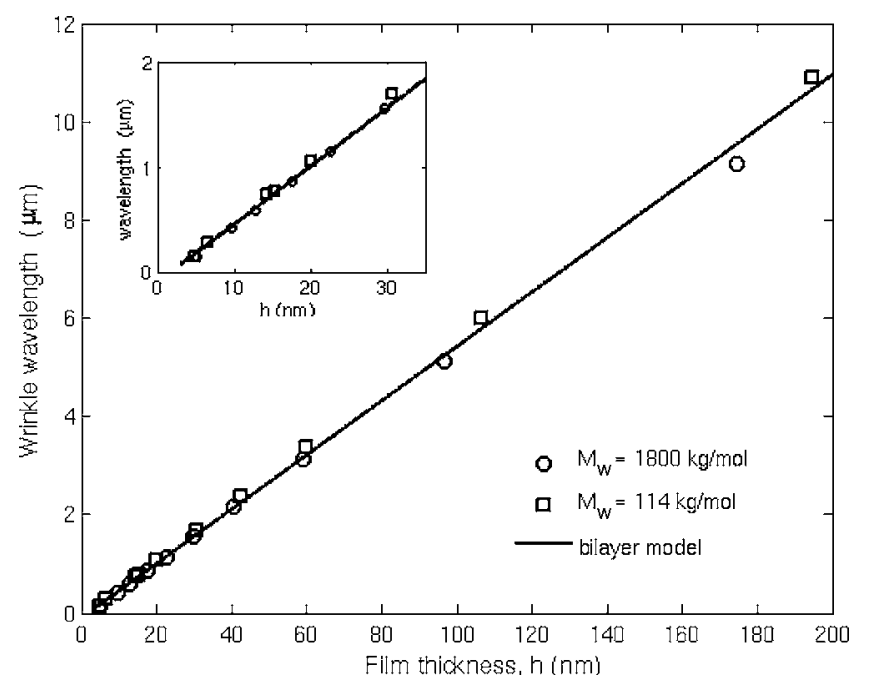

Fig. 6. Wrinkle wavelength as a function of film thickness for PS films. The inset shows the ultrathin region. 


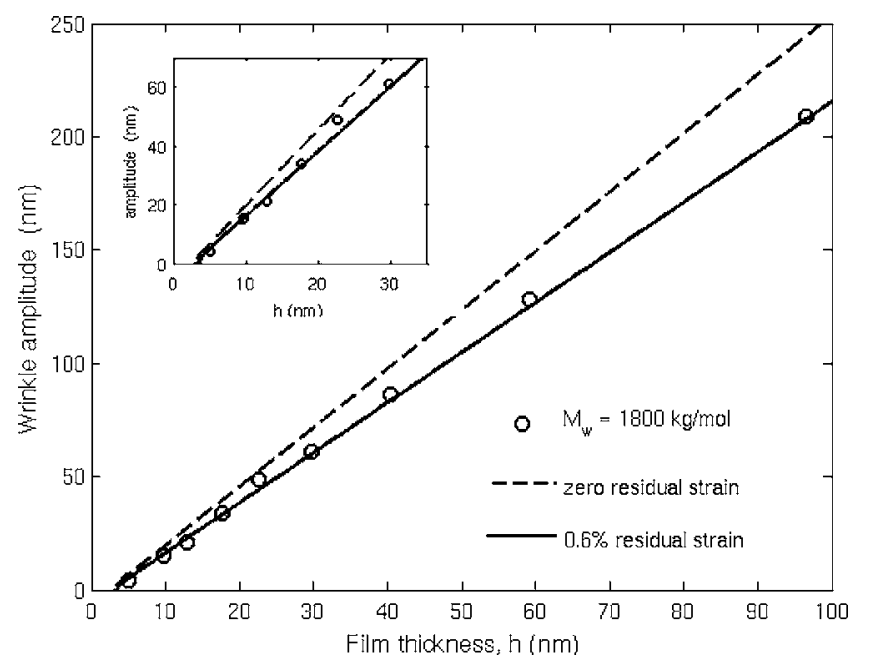

Fig. 7. Wrinkle amplitude as a function of film thickness for PS films. The inset shows the ultrathin region.

high, the stress in the film is relaxed due to wrinkling and remains below yield stress of the polymer (if applicable).

\section{Discussions}

The measured wrinkle wavelengths and amplitudes of the PS films exhibit the general trend of decreasing with decreasing film thickness (Figs. 6 and 7). Using the measured modulus for the PDMS substrate and assuming Poisson's ratio $v_{s}=0.5$, the planestrain modulus of the PS films were extracted from the wrinkle wavelengths by Eq. (2), as plotted in Fig. 8. It can be seen that, for relatively thick films $(h>30 \mathrm{~nm})$, the extracted modulus is nearly constant, independent of film thickness. For both molecular masses, the plateau modulus is about $4 \mathrm{GPa}$, which corresponds to Young's modulus $E_{f} \approx 3.6 \mathrm{GPa}$ (assuming Poisson's ratio $v_{f}=0.33$ ). This value is in good agreement with reported data for bulk PS (Fried 1995) and relatively thick PS films (Sun et al. 1994). For ultrathin PS films $(h<30 \mathrm{~nm})$, however, the deduced modulus decreases with decreasing film thickness. For the thinnest film in this study $(h \approx 5 \mathrm{~nm})$, the modulus is nearly an

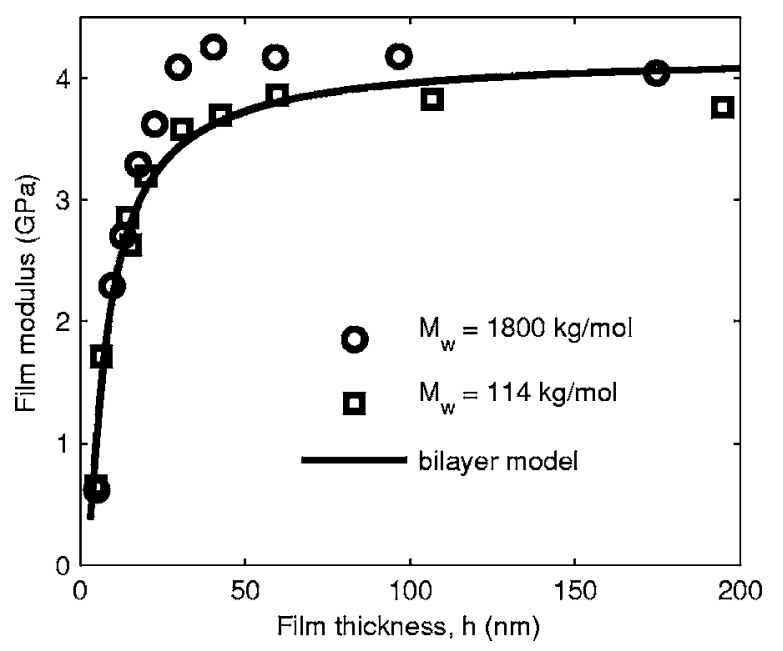

Fig. 8. Effective modulus as a function of film thickness for PS films order of magnitude lower than the plateau modulus for thick films. A similarly low modulus was estimated by Zhao et al. (2000) for $10 \mathrm{~nm}$ thick PS films probed by a wafer curvature method.

The physical origin of the thickness dependence in the deduced modulus for ultrathin PS films can be reasonably understood by using the bilayer model discussed in the section entitled "Analysis of Wrinking." According to Eq. (14), the extracted modulus in Fig. 8 is in fact the effective modulus of the bilayer for bending [Eq. (9)]. By assuming a soft surface layer $\left(\bar{E}_{f}^{*}=0.1 \mathrm{GPa}\right.$ and $\left.\delta=2 \mathrm{~nm}\right)$ and keeping the modulus constant for the rest of the film $\left(\bar{E}_{f}=4.2 \mathrm{GPa}\right)$, the effective modulus predicted by the bilayer model is plotted in Fig. 8 as a function of the total film thickness, which is in reasonable agreement with the deduced modulus. Previously, a molecular dynamics simulation (Mansfield and Theodorou 1991) showed that, within a layer of about $1.5 \mathrm{~nm}$ at the free surface of polystyrene, the mass density is lower than that in the bulk, consistent with the current assumption of a soft surface layer. For thick films, the effect of surface layer is negligible, and the effective modulus approaches the bulk modulus, independent of film thickness (plateau). For ultrathin films, the presence of the soft surface layer significantly reduces the effective modulus. The transition thickness is about one order of magnitude greater than the thickness of the surface layer. It is noted that the curve in Fig. 8 is relatively insensitive to the surface layer modulus as long as it is lower than about $0.2 \mathrm{GPa}$. Apparently, it requires more wrinkling data for film thickness in the range of $2-10 \mathrm{~nm}$ to accurately determine the surface modulus.

With the above-mentioned parameters determined for the bilayer model, the wrinkle wavelength [Eq. (14)] is plotted as a function of film thickness in Fig. 6 in comparison with the experimental data. The substrate modulus is taken to be the average of the two measured values. The agreement between the model and the data is consistently good for the entire range of film thickness. Furthermore, the predicted wrinkle amplitude [Eq. (15)] is plotted in Fig. 7 to compare with the measured amplitudes. In addition to the above parameters, the amplitude also depends on the compressive strain and the surface stress. While the applied strain in the experiments is constant $(2.5 \%)$, there is likely a residual strain in the PS films before loading. Two curves are plotted in Fig. 7: One assuming no residual strain and the other with a $0.6 \%$ tensile residual strain for all films. For both cases, the surface stress is taken to be $f=0.1 \mathrm{~N} / \mathrm{m}$. The value of surface stress has little effect on the overall curve as long as it is less than about $0.2 \mathrm{~N} / \mathrm{m}$; more amplitude data in the ultrathin region are needed for accurate determination of the surface stress. Clearly, the curve with zero residual strain overestimates the amplitudes, especially for thick films, whereas for ultrathin films $(h<30 \mathrm{~nm})$ the effect of residual strain is less important compared to the effect of surface layer. By assuming a $0.6 \%$ residual strain (i.e., total compressive strain in the film is $1.9 \%$ ), the prediction of the bilayer model is in excellent agreement with the measured wrinkle amplitudes. It should be noted that the presence of a residual strain has no effect on wrinkle wavelength. Therefore, by combining the bilayer model with a constant residual strain in the films, both the wrinkle wavelength and the amplitude are reproduced with very good agreement for thickness ranging from several nanometers to hundreds of nanometers. In addition, under the same strain, the bilayer model predicts a critical thickness at about $3 \mathrm{~nm}$. Below this thickness, the film is stable with no wrinkle; a larger compressive strain is required to cause wrinkling. 
With the consistent agreement between the model predictions and the experimental data, a set of material properties can be deduced, including elastic modulus of the film (independent of film thickness), thickness and modulus of the surface layer, surface stress, and the residual strain in the film. Among these, elastic modulus of the film $(\approx 4 \mathrm{GPa})$ can be deduced from the plateau of the effective modulus, the thickness of the surface layer $(\approx 2 \mathrm{~nm})$ can be deduced from the transition of the effective modulus, and the residual strain in the film $(\approx 0.6 \%)$ can be deduced from wrinkle amplitudes. On the other hand, accurate determination of the modulus of the surface layer $(<0.2 \mathrm{GPa})$ and the surface stress $(<0.2 \mathrm{~N} / \mathrm{m})$ require more experimental data in the ultrathin region with thickness less than $10 \mathrm{~nm}$. Nevertheless, the bilayer model provides a consistent understanding on the elastic properties and the wrinkling behavior of thin/ultrathin polymer films.

\section{Summary}

A bilayer model is proposed to account for surface effect on wrinkling of ultrathin solid films. Assuming a surface layer of finite thickness, the effects of surface properties on the critical strain, the equilibrium wavelength, and the wrinkle amplitude are discussed in comparison with conventional analysis. While surface stress has no effect on wrinkle wavelength, it can be either stabilizing or destabilizing, depending on its sign (tensile or compressive). The thickness and modulus of the surface layer lead to a size effect: the wavelength-to-thickness ratio increases (or decreases) with deceasing film thickness for ultrathin films with hard (or soft) surfaces.

Wrinkling experiments with thin polymer films were conducted for comparison to the proposed model. The measured wrinkle wavelengths and amplitudes deviate from conventional analysis for ultrathin films with thickness less than $30 \mathrm{~nm}$. The bilayer model provides a consistent understanding of the deviation, from which a set of material properties for the surface and the bulk of the polymer films are deduced from the experimental data.

A final note is addressed here as to the continuum elasticity approach taken in the present study for ultrathin films of nanometer thickness. It may be debated that the continuum approach would break down at this scale. However, since elastic modulus is simply a derivative of the free energy function at the atomic scale, continuum elasticity should be valid as long as a reasonable number of "representative" atoms or groups are available in the sample. Nevertheless, the proposed bilayer model represents one step forward from a homogeneous continuum film to a heterogeneous medium with its origin from the discreteness at the atomic scale. While the model has found its first success in wrinkling of ultrathin polymer films, its applicability for other materials remains to be investigated.

\section{Acknowledgments}

The first writer is grateful for the support by the NSF through Grant No. CMS-0547409 and by the Texas Advance Technology Program. The second and third writers gratefully acknowledge the NIST National Research Council Postdoctoral Fellowship Program.

\section{References}

Allen, H. G. (1969). Analysis and design of structural sandwich panels, Pergamon, New York.

Cahn, J. W. (1980). "Surface stress and the chemical-equilibrium of small crystals. I: The case of the isotropic surface." Acta Metall., 28(10), 1333-1338.

Cammarata, R. C. (1994). "Surface and interface stress effects in thinfilms." Prog. Surf. Sci., 46(1), 1-38.

Efimenko, K., Rackaitis, M., Manias, E., Vaziri, A., Mahadevan, L., and Genzer, J. (2005). "Nested self-similar wrinkling patterns in skins." Nat. Mater., 4(4), 293-297.

Freund, L. B. (1998). "A surface chemical potential for elastic solids." $J$. Mech. Phys. Solids, 46(10), 1835-1844.

Fried, J. R. (1995). Polymer science and technology, Prentice-Hall, Englewood Cliffs, N.J.

Gibbs, J. W. (1878). The scientific papers of J. Willard Gibbs, Vol. 1, Ox Bow, Woodbridge, Conn., 314-315 [Trans. Conn. Acad. III, 343, reprinted in 1993].

Groenewold, J. (2001). "Wrinkling of plates coupled with soft elastic media." Physica A, 298(1-2), 32-45.

Harris, A. K., Wild, P., and Stopak, D. (1980). "Silicone rubber substrata: A new wrinkle in the study of cell locomotion." Science, 208(4440), 177-179.

Harrison, C., Stafford, C. M., Zhang, W., and Karim, A. (2004). "Sinusoidal phase grating created by a tunably buckled surface." Appl. Phys. Lett., 85(18), 4016-4018.

Huang, R. (2005). "Kinetic wrinkling of an elastic film on a viscoelastic substrate." J. Mech. Phys. Solids, 53(1), 63-89.

Huang, R., and Suo, Z. (2003). "Very thin solid-on-liquid structures: The interplay of flexural rigidity, membrane force, and interfacial force." Thin Solid Films, 429(1-2), 273-281.

Huang, Z. Y., Hong, W., and Suo, Z. (2005). "Nonlinear analysis of wrinkles in films on soft elastic substrates." J. Mech. Phys. Solids, 53(9), 2101-2118.

Hutchinson, J. W., and Suo, Z. (1992). "Mixed-mode cracking in layered materials." Adv. Appl. Mech., 29, 63-191.

Iacopi, F., Brongersma, S. H., and Maex, K. (2003). "Compressive stress relaxation through buckling of a low-k polymer-thin cap layer system." Appl. Phys. Lett., 82(9), 1380-1382.

Ibach, H. (1997). "The role of surface stress in reconstruction, epitaxial growth, and stabilization of mesoscopic structures." Surf. Sci. Rep., 29(5-6), 195-263.

Im, S. H., and Huang, R. (2004). "Ratcheting-induced wrinkling of an elastic film on a metal layer under cyclic temperatures." Acta Mater, 52(12), 3707-3719.

Lacour, S. P., Wagner, S., Huang, Z. Y., and Suo, Z. (2003). "Stretchable gold conductors on elastomeric substrates." Appl. Phys. Lett., 82(15), 2404-2406.

Mansfield, K. F., and Theodorou, D. N. (1991). "Molecular dynamics simulation of a glassy polymer surface." Macromolecules, 24(23), 6283-6294.

Ortiz, M., and Gioia, G. (1997). "Delamination of compressed thin films." Adv. Appl. Mech. 33, 119-192.

Rice, J. R., and Chuang, T. J. (1981). "Energy variations in diffusive cavity growth." J. Am. Ceram. Soc., 64(1), 46-53.

Spaepen, F. (1996). "Substrate curvature resulting from the capillary forces of a liquid drop." J. Mech. Phys. Solids, 44(5), 675-681.

Stafford, C. M., et al. (2004). "A buckling-based metrology for measuring the elastic moduli of polymeric thin films." Nat. Mater., 3(8), 545550 .

Stafford, C. M., Guo, S., Chiang, M. Y. M., and Harrison, C. (2005). "Combinatorial and high-throughput measurements of thin film modulus." Rev. Sci. Instrum., 76(6), 062207.

Stafford, C. M., Vogt, B. D., Harrison, C., Julthongpiput, D., and Huang, R. (2006). "Elastic moduli of ultrathin amorphous polymer films." Macromolecules, 39(15), 5095-5099. 
Sun, L., Dutcher, J. R., Giovannini, L., Nizzoli, F., Stephens, J. R., and Ord, J. L. (1994). "Elastic and elasto-optic properties of thin-films of poly(styrene) spin-coated onto $\mathrm{Si}(001)$." J. Appl. Phys., 75(11), 7482-7488.

Watanabe, M., Shirai, H., and Hirai, T. (2002). "Wrinkled polypyrrole electrode for electroactive polymer actuators." J. Appl. Phys., 92(8),
4631-4637.

Yin, H., et al. (2003). "Buckling suppression of SiGe islands on compliant substrates." J. Appl. Phys., 94(10), 6875-6888.

Zhao, J. H., Kiene, M., Hu, C., and Ho, P. S. (2000). "Thermal stress and glass transition of ultrathin polystyrene films." Appl. Phys. Lett., 77(18), 2843-2845. 